\title{
A Systematic Review of Mobile Phone Interventions (SMS/IVR/Calls) to Improve Adherence and Retention to Antiretroviral Treatment in Low- and Middle-Income Countries
}

\author{
Binyam Afewerk Demena, PhD, ${ }^{1}$ Luis Artavia-Mora, MA, Dénis Ouedraogo, PhD, 2 \\ Boundia Alexandre Thiombiano, $\mathrm{PhD},{ }^{2}$ and Natascha Wagner, $\mathrm{PhD}^{1}$
}

\begin{abstract}
The use of mobile health technologies (mHealth) to ameliorate HIV care has considerably risen in low- and middle-income countries (LMICs) since 2010. Yet, the discrepancies in the results of accompanying studies warrant an updated and systematic consolidation of all available evidence. We report a systematic review of studies testing whether text/image messages, interactive voice response reminders, or calls promote adherence and retention to antiretroviral therapy (ART) in LMICs. We systematically compiled studies published in English until June 2018 from PubMed/Medline, Web of Science, WHO database, ProQuest Dissertations and Theses, and manual search. We used the Preferred Reporting Items for Systematic Reviews and Meta-Analyses (PRISMA) 2009 and used frequency analysis to assess reported findings. In total, we compiled 35 published articles: 27 completed studies and 8 protocols. Among the main 27 studies, 17 examine adherence, 5 retention, and 5 both measures. Results indicate that $56 \%$ report positive and statistically significantly impacts of mHealth on primary outcomes, the remaining $44 \%$ report insignificant results. While $41 \%$ of studies found a positive and significant effect for adherence, only $12 \%$ improved retention. The evidence shows ambiguous results (with high variability) about the effectiveness of mobile phone-assisted mHealth interventions to boost adherence and retention to ART. The literature also points to short follow-up periods, small samples, and limited geographical coverage. Hence, future research should focus on evaluating longer interventions with more patients spread across wider areas to address whether mHealth can be effectively used in LMICs.
\end{abstract}

Keywords: HIV/AIDS patient care, mobile health technology (mHealth), antiretroviral treatment, short message service, low- and middle-income countries

\section{Introduction}

A DHERENCE AND RETENTION to antiretroviral therapy (ART) are difficult to achieve for people living with human immunodeficiency virus (PLHIV) in particular in low- and middle-income countries (LMICs). ${ }^{1}$ PLHIV often face strong social and economic restrictions preventing them from sustaining ART. The main challenges in adherence and retention result from income losses due to lay-offs and high travel costs to medical centers that are likely to intensify discrimination, stigma, and disinformation. ${ }^{1-3}$ For instance, about $22.5 \%$ of patients in sub-Saharan Africa (SSA) discontinue ART within the first 10 months, and $56 \%$ are lost to follow-up or die within the first 2 years of treatment. ${ }^{4-6}$

To respond to this emergency, the World Health Organization (WHO) fosters the use of mobile health technologies (mHealth) in LMICs. ${ }^{7}$ Mobile phones facilitate instant communication across long distances that may encourage patients to adhere and retain to quality care. ${ }^{2,8,9}$ In particular, systems of short message services (SMS) can remind patients to take their pills, to attend refill appointments, and to retain in treatment. When SMS are used effectively, they comprise

\footnotetext{
${ }^{1}$ Institute of Social Studies, Erasmus University Rotterdam, The Hague, The Netherlands.

${ }^{2}$ Institut du Développement Rural, Université Nazi Boni (Former Université Polytechnique de Bobo-Dioulasso), Bobo-Dioulasso, Burkina Faso.

Disclaimer: The views expressed in this article are not necessarily those of 3ie or its members, or of Global Development Network (GDN).
} 
a low-barrier and low-cost intervention that yields costefficient impacts on the health of PLHIV. ${ }^{10}$ These potentially attractive benefits have stimulated numerous assessments to test whether SMS reminders can indeed improve adherence and retention to medical protocols in LMICs. ${ }^{7,11}$

Yet, there is scattered evidence about the effectiveness of SMS and other mobile phone-assisted mHealth interventions in LMICs despite their appealing features. One group of interventions shows that mobile reminders effectively promote adherence and retention to ART, ${ }^{12,13}$ but a number of studies find insignificant impacts. ${ }^{14,15}$ The high variability across findings and the considerable number of recent studies warrant the compilation of a comprehensive systematic review to organize and report the overall designs and results of SMS interventions for PLHIV in LMICs since systematic reviews are important to inform health policies and future research by establishing a global assessment of the full evidence. ${ }^{16-18}$

This article contributes to the literature by presenting an updated systematic review of primary studies about mobile phone-assisted mHealth interventions that use SMS reminders, interactive voice response (IVR) reminders, or calling to promote adherence and retention to ART in LMICs. We have also included studies in the analysis that combine SMS, IVR, or calling with other support, such as counseling. We have opted for this combination of interventions since we consider that it provides the most comprehensive overview of the scope and value added of mobile phone assisted mHealth interventions in LMICs. We focus on LMICs for two reasons: first, existing systematic reviews consolidating the evidence of mobile phone interventions on adherence and retention to ART focus on developed countries or evaluate general areas of health instead. ${ }^{19-23}$ Yet, given the high pace in the implementation and publication of recent studies, a review for LMICs becomes of paramount importance to assess the state of the art of the role of mobile phone interventions for ART. Second, PLHIV in LMICs suffer from the disease and ART in fundamentally different forms compared to patients in developed countries. Thus, this systematic review can serve as an indication for future research and public programs involving mHealth in LMICs.

\section{Materials and Methods}

We conducted a systematic literature search of studies assessing the use of mobile phone technology to promote adherence and retention to ART in LMICs. We used the Preferred Reporting Items for Systematic Reviews and MetaAnalyses 2009 (PRISMA 2009) ${ }^{24}$ when retrieving potentially relevant studies and when integrating and reporting the evidence based on inclusion and exclusion criteria ${ }^{25}$ (see complete checklist in Supplementary Appendix SA1 available online).

\section{Search strategy}

The study used a systematic web search to review primary studies on mobile phone-assisted mHealth interventions and ART in LMICs. The inclusion criteria are based on the Population Intervention Comparison Outcome (PICO) model. As shown in Table 1, we formulated the search strategy using five terms/categories. Terms within sets are connected via $\mathrm{OR}$, and terms between sets are connected using AND. All sets search the title, abstract, and keywords of articles. Fur- ther details are found in Supplementary Appendix SA2 (available online) that displays the general search used.

We screened four web-based sources and included a manual-based approach to identify studies published until June 2018. We searched for primary studies in the following databases: PubMed, Web of Science, ProQuest Dissertations and Theses, and the WHO Global Health Library. To yield database specific results, the general query was adjusted for ProQuest Dissertations and Theses and the WHO Global Health Library. In the additional manual search, we used the reference lists of the articles included in the most recent primary empirical studies.

\section{Study selection}

A two-stage selection process was chosen to determine eligibility. The first stage selected articles using title screening. Additional screening was applied using similarity of keywords in cases where articles had unclear titles. During the second-stage screening process, we reviewed abstracts and full texts (when necessary). More specifically, in the second-stage, we screened for articles that satisfied the following criteria:

1. Target outcome: adherence or retention to ART among PLHIV.

2. Empirical studies with interventions using electronic text/image messaging, IVR or call (as stand-alone or combined with other support such as counseling) for adult patients ( $>15$ years).

3. Quantitative report of (at least) one outcome from the following three: (i) adherence (self-report, pill count, electronic drug monitoring device, or pharmacy refill), (ii) retention (missed visits or patient remains on ART), and/or (iii) biological (viral load, CD4+ count, or biomarker).

4. English articles published until June 2018 in LMICs. ${ }^{26}$

\section{Data extraction}

Two reviewers (B.A.D. and L.A.-M.) independently conducted the search, the examination of eligibility, and the extraction of data. We used a standardized Microsoft Excel data template. Individually and separately, the two reviewers collected the following information about each study: publication features (year of publication, outlet/publisher, study country, web source), characteristics of the design [length of intervention, sample size, study type such as randomized controlled trial (RCT)], targeted participants, intervention (text/image/voice, language and frequency), outcome characteristics (adherence and/or retention), and intervention results (sign, magnitude, and significance of the estimates).

\section{Assessment of risk of bias}

We evaluated the methodological risk of bias (ROB) of compiled studies in accordance with the Cochrane Handbook for Systematic Reviews of Interventions. ${ }^{27}$ The process included that one reviewer (B.A.D.) evaluated the ROB and a second reviewer (L.A.-M.) independently reviewed it as well. All discrepancies were resolved with discussion and consensus. For the ROB, we report random sequence generation, allocation concealment, blinding, incomplete outcome data, 
Table 1. Terms for the Literature Search

\begin{tabular}{|c|c|c|c|}
\hline Database & Sets & Categories & Terms \\
\hline \multirow{5}{*}{$\begin{array}{l}\text { PubMed, Web of Science } \\
\text { (ISI Web of Knowledge), } \\
\text { ProQuest Dissertations } \\
\text { \& Theses, WHO Global } \\
\text { Health Library }\end{array}$} & Set 1 & Mobile phone & $\begin{array}{l}\text { Mobile phone; *mPhone; cellular phone; text message; cell } \\
\text { phone; SMS; short message service; simple message } \\
\text { service; pager; two*way electronic message* system; } \\
\text { voice call *; IVR; interactive voice * }\end{array}$ \\
\hline & Set 2 & HIV & $\begin{array}{l}\text { HIV*; *HIV; AIDS; people living with HIV*AIDS; human } \\
\text { immunodeficiency virus; PLWHA }\end{array}$ \\
\hline & Set 3 & Retention or adherence & $\begin{array}{l}\text { Adheren*; medication * adherence; retention; medication * } \\
\text { retention; CD4; biomarker; viral load }\end{array}$ \\
\hline & Set 4 & Antiretroviral therapy & $\begin{array}{l}\text { *ART; antiretroviral therapy; highly active antiretroviral } \\
\text { therapy; ARV }\end{array}$ \\
\hline & Set 5 & Region/setting & $\begin{array}{l}\text { low income } * \text {; middle income }{ }^{*} \text {; low* and middle * income } \\
\text { countr*; developing countr* }\end{array}$ \\
\hline
\end{tabular}

The asterisk (*) is added to the search terms as a placeholder for any unknown or wildcard term.

selective outcome reporting, and other sources of bias for RCTs. In addition, we included intention to treat analysis (ITT) for the incomplete outcome data of the ROB domain: defined as participants being assessed based on their initial treatment assignment (regardless of treated arm) ${ }^{28}$ We develop the ROB only for RCT studies. We do not conduct the ROB assessment for other types of interventions as there is no consensus on the quality assessment for non-RCT studies. ${ }^{27}$ RCTs were categorized at highest ROB when rated high for the random sequence generation and allocation concealment domains.

\section{Analysis of data}

Our analytical approach uses a descriptive summary of the articles included in the systematic review. To develop the analysis of the data, we performed a frequency analysis of the following characteristics: general characteristics, intervention characteristics, as well as the results/conclusions of the intervention. Although interesting, we decided not to conduct a meta-analysis given the eminent heterogeneities in the study characteristics, missing data on designs, intervention durations, and missing $p$ values in the reported outcomes.

\section{Results}

This section presents the results of the systematic review. We divide the findings into: (1) selection of studies and data extraction, (2) characteristics of studies, (3) ROB, and (4) overall assessment of the effectiveness of mHealth interventions. The main assessment examines the 27 completed studies using frequency analysis.

\section{Selection of studies and data extraction}

The selection and extraction of data were developed by two independent and separate reviewers (B.A.D. and L.A.-M.). Following the two-stage selection process, the first stage identified a total of 195 studies using title screening. Most first-stage studies were irrelevant as we used an inclusive search query that resulted in a large set of false hits. Thus, we conducted the second-stage screening process by reviewing abstracts and full texts (when necessary). Figure 1 shows the flowchart and number of studies identified and selected across the two-stage selection process.

The screening of titles and abstracts in the second stage excluded 102 articles that were selected in the first stage. The duplication of studies was also common across electronic databases, so only 75 peer-reviewed articles remained in our systematic review after removing duplicates. In addition, we dropped another 49 studies after the careful assessment of full texts. Supplementary Appendix SA3 (available online) provides a complete record of excluded studies with a justification for their exclusion. The subsequent manual search identified 27 additional studies through citation tracking. After review of the abstracts and full texts, only nine more articles were added as they matched the inclusion criteria. Table 2 presents the number of studies retrieved and selected per database. At the end of the process, we identified and selected a final sample of 27 completed studies and 8 protocols. Table 3 presents detailed information of the included articles. Note that the eight protocols are excluded from our analysis as we focus on after-intervention estimates.

\section{Characteristics of the studies}

In terms of scope, the 27 completed studies were carried out in Africa, Asia, and Latin America. Articles on Africa have the highest share reaching about $63 \%$. Twelve studies were performed in Cameroon, Kenya, South Africa, and Uganda (three in each country), while Mozambique and Swaziland account for four articles (two each), and one was done in Nigeria. This strong focus on Africa is consistent with the high prevalence of HIV on the continent; more than 3.9 million children and adults in SSA undergo ART and the management of the HIV epidemic is a priority on the international health agenda. ${ }^{29,30}$ Meanwhile, Asian countries accounted for $33 \%$ of the 27 articles: 8 articles tested interventions in China and India (4 in each country) and a single article shows results for Pakistan. Only one study was conducted in Latin America (Brazil).

The two most common interventions investigate the impact of: (1) standardized SMS messages (text, image, or both), or (2) IVR reminders. Nearly $90 \%(n=24)$ of the studies investigate standardized SMS messages, of which four are combined with IVR, calling or counseling. One study in China combined calls with bimonthly nurse home visits. ${ }^{31}$ Ownership of a mobile phone was not an eligibility criterion in two RCTs that provided mobile phones (Rural Kenya and South India). ${ }^{13,14}$ Only two of the RCTs assessed IVR alone with one of them finding no statistically significant impact. Moreover, we find high variation in terms of the time period, during which patients received the intervention. The 


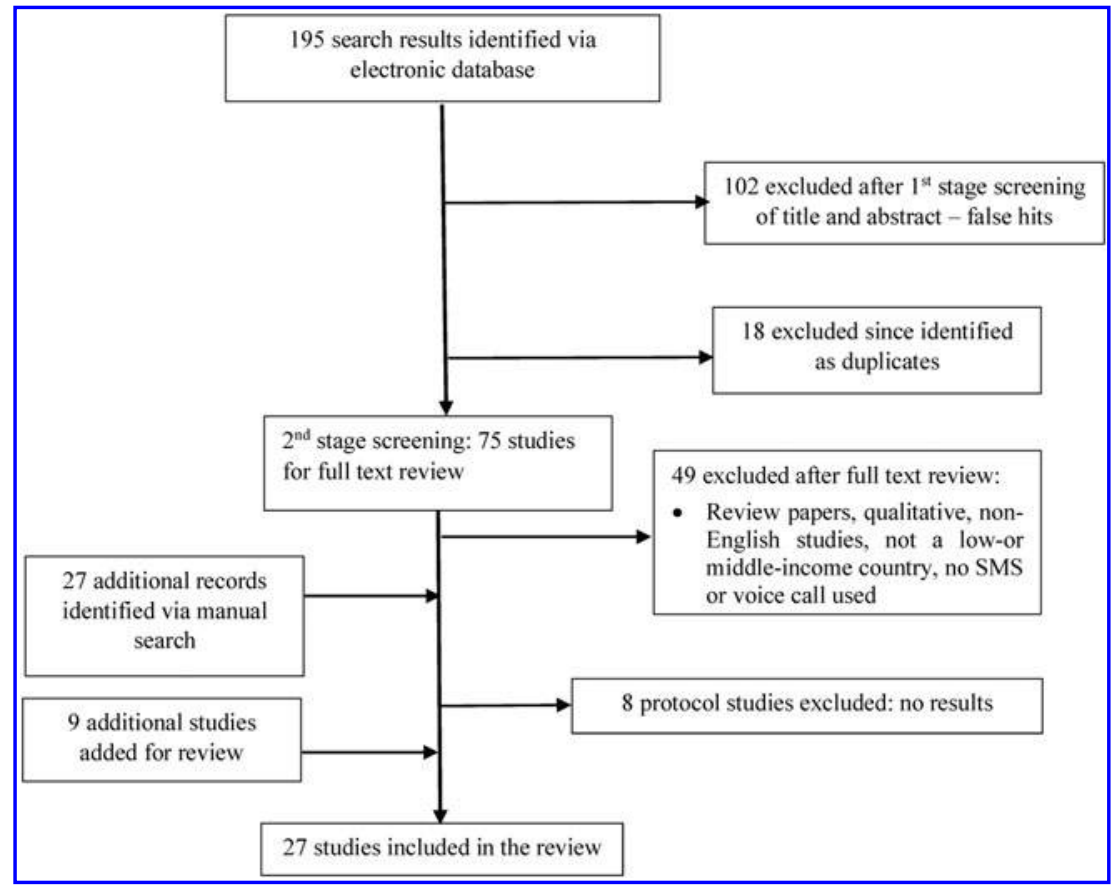

FIG. 1. Flowchart of literature selection process. minimum time that patients received messages was 1 month (implemented in three studies in North Western Cameroon, Southern India, and Indian West Bengal). ${ }^{32-34}$ A maximum period of 24 months was used for a study on health care delivery in South India. ${ }^{14}$ The average evaluation period was 9 months. Assessing the RCT studies separately, we find a similar picture of short-term interventions except for one study. ${ }^{14}$ In terms of evaluation designs, $74 \%$ are RCTs $(n=20), 7.4 \%$ are cohort studies $(n=2)$, and $7.4 \%$ use a quasiexperimental design $(n=2)$. The remaining $11.2 \%$ include controlled studies $(n=3)$ that use before-after, cross sectional, or pilot designs.

The messages were formulated to remind patients of medication, appointments, education, and/or counseling offers. The content was often generic (10 of 27 studies). This approach is supported with evidence from Peru examining the perceptions of PLHIV about message content and suggesting that concise, general messages are the preferred choice. ${ }^{35}$ In contrast, in three reviewed studies, message content was personalized, including the name of the recipient. In 14 of the 27 studies, the authors did not explicitly specify the content of the messages. Several examples of message content are presented in Fig. 2. Regarding the language of the messages, in 17 studies the

Table 2. Literature Search Results by Database

\begin{tabular}{lcc}
\hline Database & $\begin{array}{c}\text { Results } \\
\text { retrieved }\end{array}$ & $\begin{array}{c}\text { Results } \\
\text { selected }\end{array}$ \\
\hline PubMed & 115 & $16(3)$ \\
Web of Science (ISI Web & 24 & 2 \\
$\quad$ of Knowledge) & 15 & 0 \\
ProQuest Dissertations \& Theses & 41 & $1(4)$ \\
WHO Global Health Library & 27 & $8(1)$ \\
Hand-searching/citation tracing & 222 & $27(8)$ \\
Total & & \\
\hline
\end{tabular}

Figures in parentheses stand for protocol studies. message reminders were sent in the local or preferred language of the participant. This finding builds on earlier research showing that illiteracy is negatively associated with impacts of message reminders. ${ }^{36}$

The geographical coverage of the interventions is not uniform across studies but tends to be restricted. Nearly one in three studies focuses on urban areas. It was only in Africa that also interventions in rural areas were studied ( 7 of the 17 studies). The 10 studies from Asia and Latin America focus merely on urban areas. No study uses a nationally representative sampling strategy in the evaluation design. But as complementary results, we identify two published protocols that aim at addressing this weakness. First, L'Engle et al. ${ }^{37}$ published a protocol to conduct a 3-year intervention with 1600 patients in 5-regions of Ghana. But this study was suspended before launching the intervention due to uncertainties about the success of implementation. Second, there is one published protocol implementing a nationwide study with a representative sample in Burkina Faso. ${ }^{38}$ This study rests on a sample of more than 3800 patients in a fivearm RCT over a 2-year period.

Our findings show high variability in the number of participants and evaluation methods but similarities in the age of

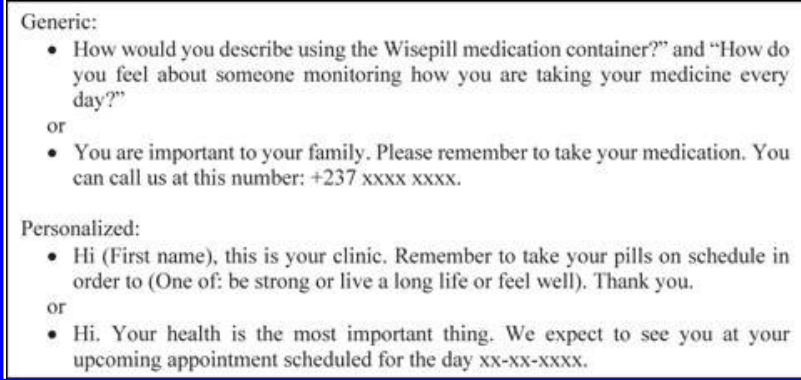

FIG. 2. Examples of message reminders sent to patients. 
the target group. The largest study included 2255 participants, but about $74 \%$ of the studies had less than 500 participants with a median of 196 . Recent studies argue that research about adherence and retention to ART require large sample sizes as well as sustained periods of the mHealth intervention. ${ }^{6,38}$ However, more than half of the studies included in the review only assesses short-run impacts in small samples. There are three exceptions (studies in Mozambique, South Africa, and Swaziland) that on average assess 2152 patients over a 12 months period. ${ }^{39-41}$ Across the 20 RCTs, only 2 use a large sample and 9 have less than the median sample size of the 27 studies selected in the present review. In contrast, the review finds similarities across studies in the minimum age of the participants. Most interventions targeted participants older than 18 years, who were relatively ARTnaive (no more than 3 months), and in possession of a mobile phone. Two studies included participants from the age of 15 years (South Africa and Uganda). ${ }^{42,43}$ In five articles, the age of the participants was not specified.

Regarding publication dates, we find studies from developed countries since the early 2000s. ${ }^{9}$ For LMICs, the first two studies for China and Kenya were published in $2010 .^{31,44}$ Both studies were performed in three different HIV clinics with different economic conditions. In Kenya, a total of 538 patients were randomly assigned to a control and treatment group, while the intervention in China only included 116 patients. Over the years, the number of interventions has been increasing (review details in Table 3 ). The median article appeared in 2014 and most articles were published in 2017.

\section{$R O B$ in the identified studies}

Figure 3 presents a summary of the ROB items for the RCTs. Exactly $85 \%$ of the 20 articles that use RCTs were rated having low risk of random sequence generation bias. Most studies applied computer-assisted randomization or minimization. The remaining three studies $(15 \%)$ had unclear risk of random sequence generation. For allocation concealment, we find a similar pattern: $80 \%$ of the studies were at low risk, while the rest at an unclear level. Importantly, none of the studies was rated at high risk in these two domains. However, blinding of participants, medical staff, and assessors was not done across studies. Performance bias was rated at high risk for most studies (85\%). Similar results are observed for detection bias; yet, four studies had a low bias in this domain. The other domains of ROB were rated at low risk for $70-95 \%$ of the studies.

Detailed ROB assessments for each reviewed study are provided in Supplementary Appendix SA4 (available online); 13 studies were rated with low risk in all domains except for blinding, which was rated at high risk. For the remaining seven RCTs, at least one item of selection bias was unclear. $^{31,32,45-47}$ Two studies did not show ITT estimates ${ }^{12,47}$ and the estimation method was unclear in one other study. ${ }^{46}$ This latter study was also rated with high attrition bias. No other study obtained a high-risk score in attrition bias. Moreover, one study scored high in risk of selective reporting bias $^{48}$ whereas five studies showed an unclear bias. ${ }^{12,32,45,49,50}$ Other dimensions of bias, such as conflict of interest or funding, were unclear in two studies, ${ }^{32,42}$ while all the remaining studies had low ROB results.

\section{Did SMS or IVR promote adherence and retention?}

Of the 27 published studies included in our systematic review, 17 (63\%) examined the utilization of mobile technology (SMS, voice or both) on adherence while only $5(18.5 \%)$ assessed retention. Another 5 (18.5\%) of the studies evaluate both adherence and retention to ART. Adherence was typically defined using self-reports, pill counts, an electronic medication event monitoring system, and/or suppression of viral load. In turn, retention was measured as loss to follow-up (with and without identifiable cause) or the rate of scheduled visits missed.

In total, 56\% of the studies found that mobile technology had positive and statistically significant impacts on adherence and retention $(n=15)$. In contrast, $44 \%$ report that using mobile phones was not effective to ameliorate any of these two outcomes in PLHIV $(n=12)$. If we examine each outcome separately, for adherence, $41 \%$ find positive and significant results and $28 \%$ insignificant impacts, while $12 \%$ of the studies reported positive retention effects and 19\% insignificant effects. Note that we have 5 studies that reported both adherence and retention resulting in 10 outcomes plus the 22 studies that reported either retention or adherence. This results in a total of 32 possible outcomes: 22 adherence and 10 retention outcomes, of which 13 and 4 found positive and statistically significant effects, respectively. Overall, the systematic review shows that only about one in two studies has achieved the objective of measurable improvements in ART revealing high variability across studies and outcomes. The findings further suggest that mobile phone-assisted mHealth has higher impacts on measures of adherence than retention. Table 4 presents the results for the main outcomes of the systematic review. Since across studies, a host of different outcome indicators has been chosen, we can only speculate that identifiable impacts might depend, at least to some extent, on the choice of outcome.

Furthermore, the previous literature argues that the lack of positive and statistically significant impacts is linked to the small number of completed RCTs ${ }^{51}$ Notice, however, that RCT designs represent the dominant evaluation method in our review (74\%, 20 of 27 studies). Thus, after restricting our sample to RCT studies as a qualitative robustness test, we find that $60 \%$ (12 of 20) show a positive and statistically significant increase of adherence and/or retention to ART. This result is consistent with the findings obtained using the whole sample. There is only a 4-percentage point difference in favor of the randomized studies.

\section{Discussion}

This article conducts a systematic review of mobile phoneassisted mHealth interventions designed to improve adherence and retention to ART in LMICs. Informed by the PRISMA 2009 Guidelines, we systematically included studies from PubMed/Medline, Web of Science, WHO database, ProQuest Dissertations and Theses, and manual search published in English language until June 2018 (inclusive). We used frequency analysis of the collected data to systematically examine the results of the reported outcomes. We selected a total of 35 studies divided between 27 completed evaluations with published results and 8 protocols of ongoing interventions. Among the 27 finished studies 17 (63\%) investigated adherence to ART, 5 studies (18.5\%) focused on retention in care, and 5 (18.5\%) jointly assessed adherence and retention; in terms of the geographical 

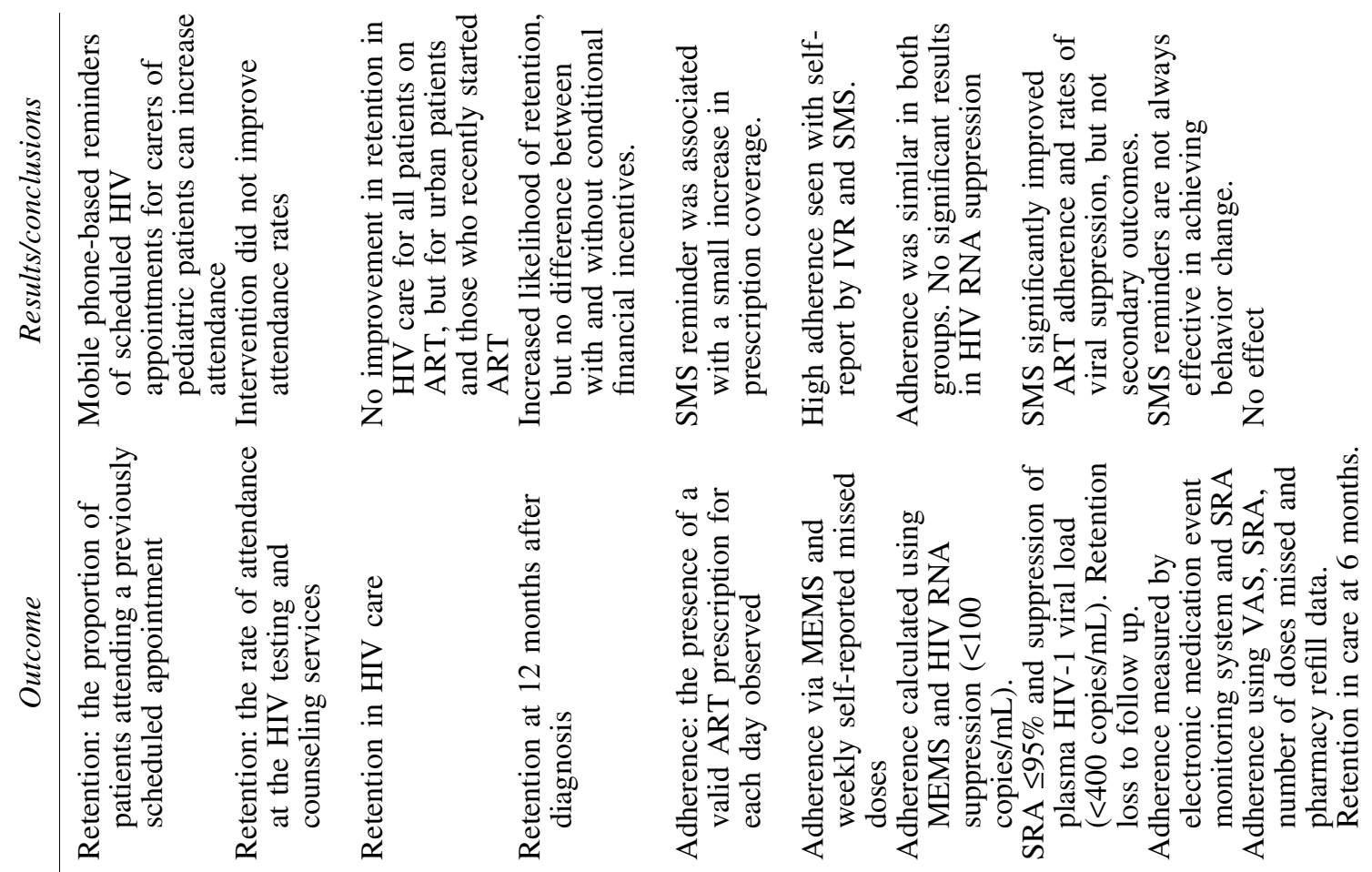

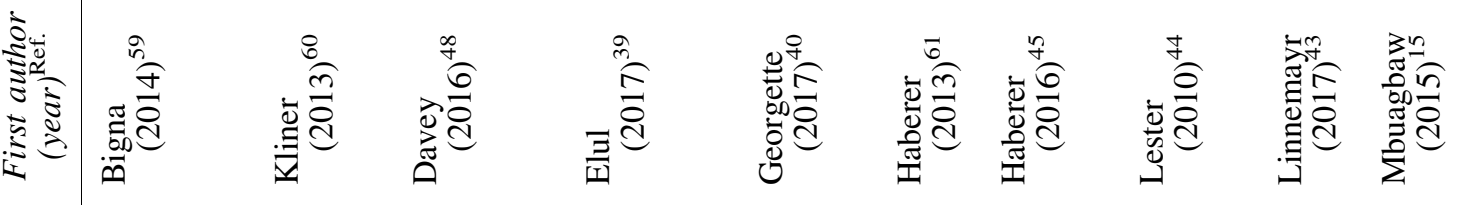
$\dot{z}-\quad$ n $\quad$ क 


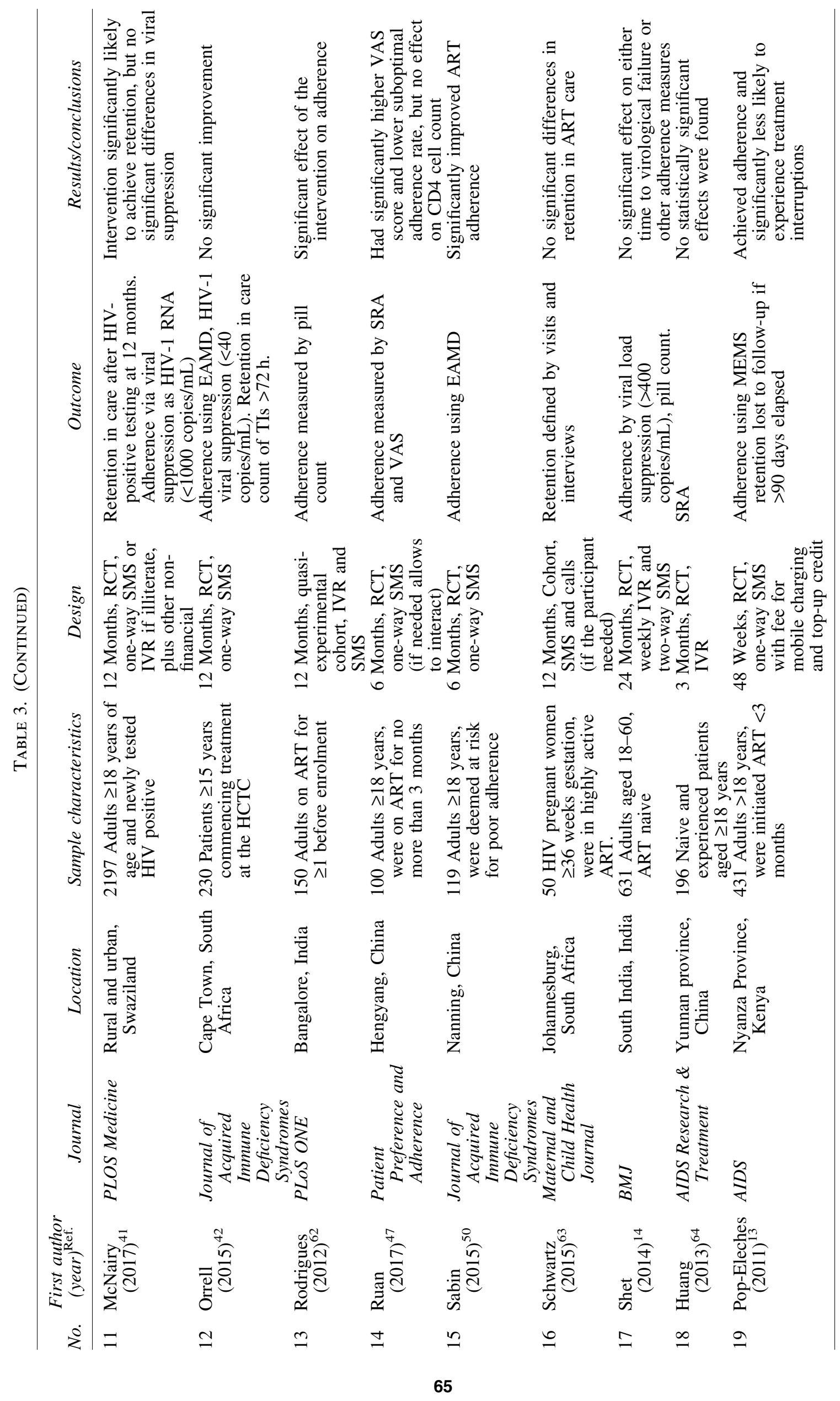




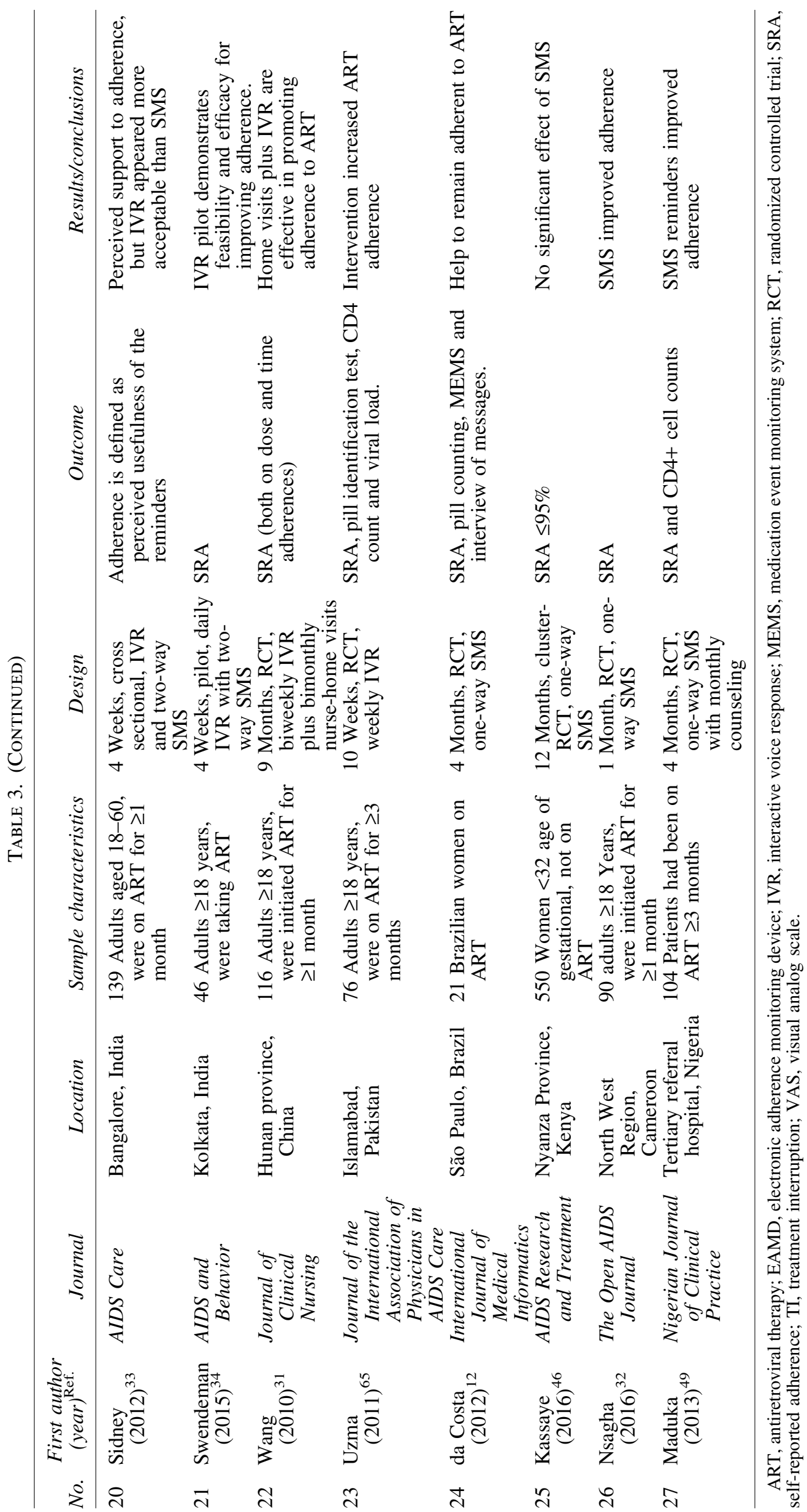




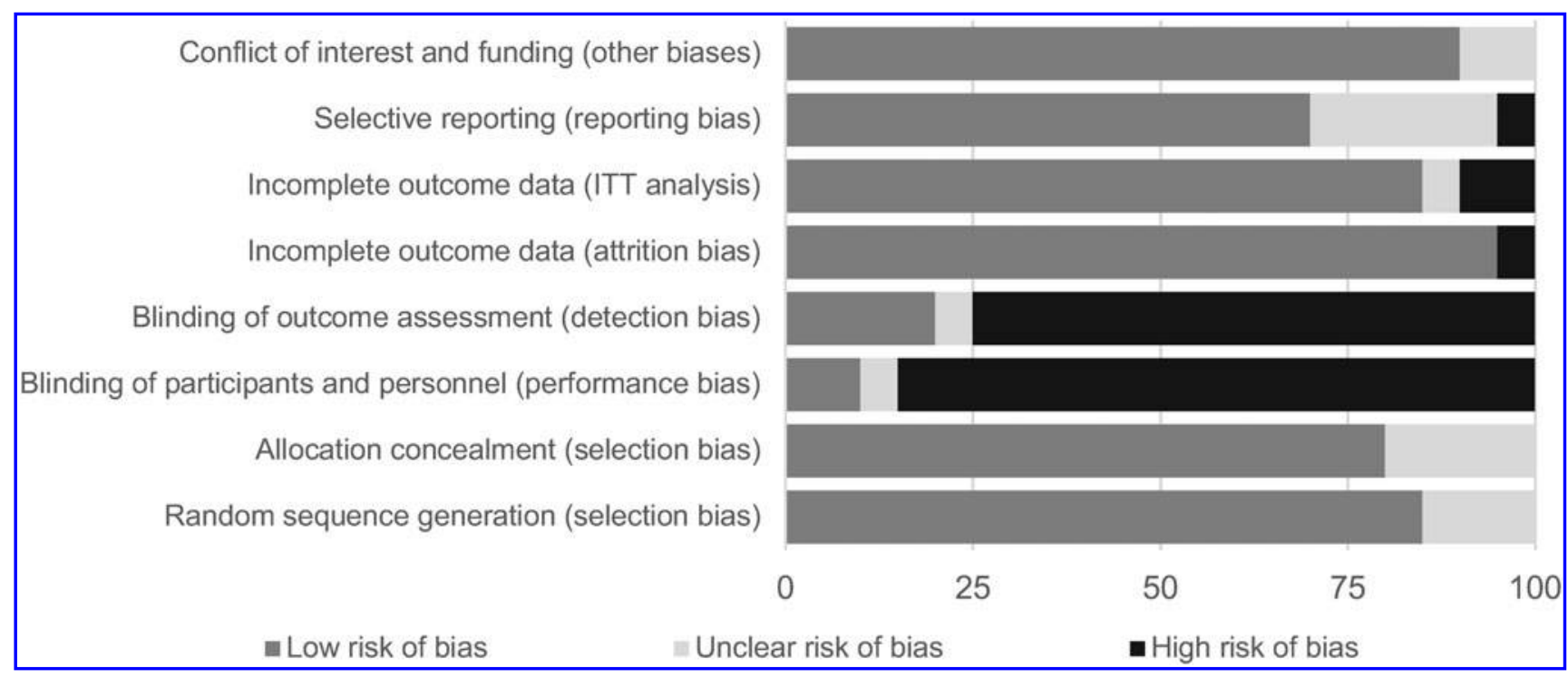

FIG. 3. Summary of risk of bias items across included studies (\%).

distribution, 17 studies were carried out in SSA, 9 in Asia, and only 1 in Latin America.

We show that $56 \%$ of the 27 completed studies indicate that mobile technology positively and significantly impacts adherence and retention to ART. These findings are robust to excluding nonrandomized studies, which shows that results are robust to design or program implementation. By outcome variable, mobile phone-assisted mHealth is effective in increasing adherence (41\%) compared to retention (12\%). Thus, the evidence suggests that identifiable impacts of SMS, IVR, and call for ART might be found only along some dimensions and consequently also depend on the choice of outcome variable. We find high variability in the characteristics of the interventions. Moreover, most studies comprised short analysis periods and small samples covering highly restricted geographical areas. A serious downside is that not a single study achieves national representativeness. Hence, the reviewed evidence is still rather ambiguous about the effectiveness of mobile phone-assisted mHealth interventions for PLHIV in LMICs. Future research in the area must expand the study duration and sample size to achieve better representativeness and gauge possible (new) dimensions of impact. An existing effort is presented in the published protocol by Wagner et al. for Burkina Faso. ${ }^{38}$

\section{Does our systematic review agrees or disagrees} with the wider evidence?

Comparing the results from the present systematic review with previous work confirms both, namely that (1) the impact of SMS and IVR interventions is overall ambiguous, and that
(2) there is only scarce evidence of systematic reviews for LMICs. First, a Cochrane review pooling two studies for Kenya finds that SMS messages did not reduce the risk for nonadherence. ${ }^{19}$ These two studies are also included in our systematic review. ${ }^{13,44}$ Second, Govindasamy et al. ${ }^{21}$ provide a review of 24 studies published between 2004 and 2013 about retention in pre-ART care and initiation of ART from various interventions (not only mHealth) in LMICs. Although that review shows that several interventions produced favorable outcomes in observational cohort studies, it has two caveats compared to our systematic review: (1) it suffers from unclear ROB, and (2) an incomprehensive selection of studies published until 2013. We have included 12 studies published until 2013, however, none of these studies was included in the review by Govindasamy et al. ${ }^{21}$

Kanters et al. ${ }^{52}$ show in a meta-analytical research that multiple interventions are significantly better than single interventions to improve adherence. Compared to standard care alone, SMS interventions had positive impacts globally and when only considering LMICs. Overall, the consolidated evidence suggests that while (multiple) interventions can improve adherence and viral suppression, their impact is modest, fades over time, and differs across countries. In similar vein, recent qualitative evidence from India suggests that mobile phone-based counseling for HIV-positive women is well perceived. ${ }^{53}$ It is argued that calls might be more acceptable in accompanying ART than SMS.

In sum, our review combines evidence from existing reviews with new studies that were not considered in previous systematic reviews on LMICs. We close an evidence gap as

Table 4. Classification of Outcomes from the Reviewed Articles

\begin{tabular}{lccr}
\hline Outcome & Insignificant impact & Significant positive impact & Total no. of articles (\%) \\
\hline Retention & $3[1]$ & $2[2]$ & $5(18.5 \%) ;[3(15 \%)]$ \\
Adherence & $6[4]$ & $11[8]$ & $17(63 \%) ;[12(60 \%)]$ \\
Retention and adherence & $3[3]$ & $2[2]$ & $5(18.5 \%) ;[5(25 \%)]$ \\
Total & $12[8]$ & $15[12]$ & $27(100 \%) ;[20(100)]$ \\
\hline
\end{tabular}

Figures in square brackets stand for RCTs.

RCT, randomized controlled trial. 
there is scarce evidence rigorously compiling the existing primary studies on mobile phone-assisted mHealth interventions for PLHIV outside developed countries.

In addition, our results can be more readily compared to current findings for developed countries. A recent review of 35 studies carried out in developed countries, including 13 RCTs from the United States, found unclear results for interventions evaluating a wider scope of sexually transmitted diseases and health management (prevention, detection, treatment, and knowledge). ${ }^{28}$ Another systematic review of 34 studies (mainly United States, $n=15$ ) indicates that text message interventions improve adherence rates, but this review includes different methods of evaluation and diseases (i.e., asthma, diabetes, heart problems, and schizophrenia). ${ }^{54}$ It evaluated only six studies involving patients in ART. Similarly, Chaiyachati et al., ${ }^{9}$ in a review composed of 20 studies mainly from North America, found high variability in the effectiveness of a combination of interventions that include (1) adherence reminders, (2) cognitive-behavioral interventions, (3) education, (4) treatment supporters, and (5) directly observed therapy. Although these five interventions can improve adherence to ART, impacts are not coherent across settings. ${ }^{9}$ Similarly, Guy et al. ${ }^{20}$ find that SMS reminders reduce nonattendance rates and improve medical attendance in a review of 18 studies (mostly) in European countries that were published between 2006 and 2010.

Our systematic review differs in three fundamental points from another systematic review of 28 interventions showing that mHealth provides positive impacts on HIV-outcomes. ${ }^{55}$ First, our research focuses exclusively on LMICs. In contrast, Cooper et al. ${ }^{55}$ jointly assess evidence from high- and lowincome settings. Most of our primary studies originate from interventions in Africa (63\%), while the majority of their studies are from North America (56\%). Second and contrary to the earlier review, our review assesses not only adherence but also retention. Third, we extend the timeframe to June 2018 and thus expand it from the earlier end date of October 2016.

After a careful analysis of the existing literature we found that available reviews are restricted to specific types of program implementation, ${ }^{28,54}$ or include only one of the two primary outcomes. ${ }^{9,20}$ Moreover, as outlined above, existing studies about LMICs tend to be limited in scope. ${ }^{19,21}$ Our review differs from previous work as we include all quantitative study designs and the two most commonly assessed outcomes (retention and adherence) of ART interventions.

\section{Limitations}

Our review has three main limitations. First, we only compile studies written in English due to challenges reviewing studies in other languages. Including articles in other languages may provide a more holistic representation of mHealth activities. Notably, countries speaking other languages may show different responses to mobile phone interventions. Yet, since English is the main scientific language, we expect that we covered all major studies. Second, we exclusively included studies from LMICs. We took that decision because the health care system and situation of PLHIV are very different in LMICs compared to high-income countries, and we included a short comparison between our study and related systematic reviews for developed countries. We are aware that recently three innovative $\mathrm{mHealth}$ interventions for
PLHIV have been implemented in the United States using (1) a smartphone app to connect patients to clinic staff and educational resources, ${ }^{56}$ (2) social media, ${ }_{58}$ and (3) daily messages plus counseling. ${ }^{58}$

Finally, we did not conduct a meta-analysis because of the large heterogeneity across studies. We identified many different study designs, intervention types, durations, various measures for similar outcomes, and, in particular, we found that numerous $p$-values for the reported outcomes were missing.

\section{Conclusions}

We systematically review studies testing mHealth interventions (SMS, IVR, calls) on health outcomes of PLHIV in LMICs that were published until June 2018. Previous systematic reviews concentrate on developed countries, ${ }^{54}$ are restricted to specific types of program implementation, ${ }^{28,54}$ are limited to RCT designs, ${ }^{19}$ or include only one of our two primary outcomes. ${ }^{9,20}$ There is scarce evidence rigorously compiling the existing primary studies on mobile phoneassisted mHealth interventions outside developed countries. To the best of our knowledge, only one review was conducted for LMICs assessing retention in pre-ART care and initiation of ART from various interventions (not only mHealth) up to the year 2013. ${ }^{21}$ Thus, our study expands the timeframe and results by including the most recent studies for LMICs (until June 2018) and also covers a larger set of outcomes and a number of new studies that are not considered in previous systematic reviews on LMICs.

The consolidated evidence shows ambiguous results (with high variability) about the effectiveness of mobile phoneassisted mHealth to boost adherence and retention to ART. The review also points to short follow-up periods, small samples, and narrow geographical areas, although we highlight the current intervention in Burkina Faso. ${ }^{38}$ Hence, evaluating longer interventions and larger number of patients across wider national spaces should be a priority of future research. The latter may resolve whether mobile phone-assisted mHealth interventions can be effectively used for PLHIV in LMICs.

\section{Author Disclosure Statement}

No competing financial interests exist.

\section{Funding Information}

Research discussed in this publication has been funded by the International Initiative for Impact Evaluation, Inc. (3ie) through the Global Development Network (GDN).

\section{Supplementary Materials}

Supplementary Appendix SA1

Supplementary Appendix SA2

Supplementary Appendix SA3

Supplementary Appendix SA4

\section{References}

1. Scanlon ML, Vreeman RC. Current strategies for improving access and adherence to antiretroviral therapies in resource-limited settings. HIV AIDS (Auckl) 2013;5:1-17.

2. Lester RT, Gelmon L, Plummer FA. Cell phones: Tightening the communication gap in resource-limited antiretroviral programmes? AIDS 2006;20:2242-2244. 
3. Mills EJ, Nachega JB, Buchan I, et al. Adherence to antiretroviral therapy in sub-Saharan Africa and North America: A meta-analysis. JAMA 2006;296:679-690.

4. Fox MP, Rosen S. Patient retention in antiretroviral therapy programs up to three years on treatment in sub-Saharan Africa, 2007-2009: Systematic review. Trop Med Int Health 2010;Suppl 1:1-15.

5. Rosen S, Fox MP, Gill CJ. Patient retention in antiretroviral therapy programs in sub-Saharan Africa: A systematic review. PLoS Med 2007;4:e298.

6. Rosen S, Fox MP. Retention in HIV care between testing and treatment in sub-Saharan Africa: A systematic review. PLoS Med 2011;8:e1001056.

7. WHO. mHealth: New Horizons for Health Through Mobile Technologies. Geneva, Switzerland: World Health Organization; 2011

8. Coomes CM, Lewis MA, Uhrig JD, Furberg RD, Harris JL, Bann CM. Beyond reminders: A conceptual framework for using short message service to promote prevention and improve healthcare quality and clinical outcomes for people living with HIV. AIDS Care 2012;24:348-357.

9. Chaiyachati KH, Ogbuoji O, Price M, Suthar AB, Negussie EK, Bärnighausen T. Interventions to improve adherence to antiretroviral therapy: A rapid systematic review. AIDS 2014;28:S187-S204.

10. Lester R, Karanja S. Mobile phones: Exceptional tools for HIV/AIDS, health, and crisis management. Lancet Infect Dis 2008;8:738-739.

11. Xiong K. Mobile technology for monitoring and evaluation and health information systems in low-to middle-income countries. MEASURE Evaluation Special Report 2015:11-16. Available at: https://www.measureevaluation.org/resources/ publications/sr-15-116 (Last accessed January 22, 2020).

12. da Costa TM, Barbosa BJP, de Costa DAG, et al. Results of a randomized controlled trial to assess the effects of a mobile SMS-based intervention on treatment adherence in HIV/AIDS-infected Brazilian women and impressions and satisfaction with respect to incoming messages. Int J Med Inform 2012;81:257-269.*

13. Pop-Eleches C, Thirumurthy H, Habyarimana JP, et al. Mobile phone technologies improve adherence to antiretroviral treatment in a resource-limited setting: A randomized controlled trial of text message reminders. AIDS (London, England) 2011;25:825*

14. Shet A, De Costa A, Kumarasamy N, et al. Effect of mobile telephone reminders on treatment outcome in HIV: Evidence from a randomised controlled trial in India. BMJ 2014;349:g5978.*

15. Mbuagbaw L, Thabane L, Ongolo-Zogo P, et al. The Cameroon Mobile Phone SMS (CAMPS) trial: A randomized trial of text messaging versus usual care for adherence to antiretroviral therapy. PLoS One 2015;7:e46909.*

16. Bambra C, Joyce KE, Bellis MA, et al. Reducing health inequalities in priority public health conditions: Using rapid review to develop proposals for evidence-based policy. J Public Health (Oxf) 2010;32:496-505.

17. Greenhalgh T, Peacock R. Effectiveness and efficiency of search methods in systematic reviews of complex evidence: Audit of primary sources. BMJ 2005;331:1064-1065.

18. Khangura S, Konnyu K, Cushman R, Grimshaw J, Moher D. Evidence summaries: The evolution of a rapid review approach. Syst Rev 2012;1:10.
19. Horvath T, Azman H, Kennedy GE, Rutherford GW. Mobile phone text messaging for promoting adherence to antiretroviral therapy in patients with HIV infection. Cochrane Database Syst Rev 2012:CD009756.

20. Guy R, Hocking J, Wand H, Stott S, Ali H, Kaldor J. How effective are short message service reminders at increasing clinic attendance? A meta-analysis and systematic review. Health Serv Res 2012;47:614-632.

21. Govindasamy D, Meghiz J, Negussi EK, Baggaley RC, Ford N, Kranzer K. Interventions to improve or facilitate linkage to or retention in pre-ART (HIV) care and initiation of ART in low-and middle-income settings-A systematic review. J Int AIDS Soc 2014;17:19032.

22. Laisaar KT, Raag M, Rosenthal M, Uusküla A. Behavioral interventions to reduce sexual risk behavior in adults with HIV/AIDS receiving HIV care: A systematic review. AIDS Patient Care STDs 2015;29:288-298.

23. O'Brien N, Hong QN, Law S, et al. Health system features that enhance access to comprehensive primary care for women living with HIV in high-income settings: A systematic mixed studies review. AIDS Patient Care STDs 2018;32:129-148.

24. Liberati A, Altman DG, Tetzlaff J, et al. The PRISMA statement for reporting systematic reviews and metaanalyses of studies that evaluate health care interventions: Explanation and elaboration. PLoS Med 2009;6:e1000100.

25. Moher D, Liberati A, Tetzlaff J, Altman DG. The PRISMA Group. Preferred reporting items for systematic reviews and meta-analyses: The PRISMA Statement. PLoS Med 2009;6:e1000097.

26. World Bank. Data and Statistics. Country classification. [Internet]. 2016. [cited June 3, 2018]. Available at: http://blogs .worldbank.org/opendata/new-country-classifications-2016 (Last accessed June 3, 2018).

27. Higgins JPT, Altman DG, Sterne JAC. Chapter 8: Assessing risk of bias in included studies. In: Cochran Handbook for Systematic Reviews of Interventions: 5.2. The Cochrane Collaboration; 2011. JPT Higgins and S Green, eds. Available at: www.handbook.cochrane.org (Last accessed January 22, 2020).

28. Taylor D, Lunny C, Lolić P, et al. Effectiveness of text messaging interventions on prevention, detection, treatment, and knowledge outcomes for sexually transmitted infections (STIs)/HIV: A systematic review and metaanalysis. Syst Rev 2019;8:12.

29. UNAIDS: Global AIDS Update. 2018. Miles to Go: Closing Gaps, Breaking Barriers, Righting Injustices. Geneva, Switzerland. Available at: http://www.unaids.org/sites/default/files/ media_asset/miles-to-go_en.pdf (Last accessed December 12, 2018).

30. World Bank. Data and Statistics. Country classification. [Internet]. 2018. [cited June 3, 2018]. Available at: http:// data.worldbank.org/about/countryclassifications/countryand-lending-groups (Last accessed June 3, 2018).

31. Wang H, Zhou J, Huang L, Li X, Fennie KP, Williams AB. Effects of nurse-delivered home visits combined with telephone calls on medication adherence and quality of life in HIV-infected heroin users in Hunan of China. J Clin Nurs 2010;19:380-388.*

32. Nsagha DS, Lange I, Fon PN, Assob JC, Tanue EA. A randomized controlled trial on the usefulness of mobile text phone messages to improve the quality of care of HIV

*Primary studies in the systematic review. 
and AIDS patients in Cameroon. Open AIDS J 2016;10: 93.*

33. Sidney K, Antony J, Rodrigues R, et al. Supporting patient adherence to antiretrovirals using mobile phone reminders: Patient responses from South India. AIDS Care 2012;24:612-617B.*

34. Swendeman D, Jana S, Ray P, Mindry D, Das M, Bhakta B. Development and pilot testing of daily interactive voice response (IVR) calls to support antiretroviral adherence in India: A mixed-methods pilot study. AIDS Behav 2015;19: $142-155 *$ *

35. Curioso WH, Quistberg DA, Cabello R, et al. "It's time for your life": How should we remind patients to take medicines using short text messages? AMIA Annu Symp Proc 2009:129-133.

36. Kaplan WA. Can the ubiquitous power of mobile phones be used to improve health outcomes in developing countries? Global Health 2006;2:9.

37. L'Engle KL, Green K, Succop SM, Laar A, Wambugu S. Scaled-Up mobile phone intervention for HIV care and treatment: Protocol for a facility randomized controlled trial. JMIR Res Protoc 2015;4:e11.

38. Wagner N, Ouedraogo D, Artavia-Mora L, Bedi A, Thiombiano BA. Protocol for a randomized controlled trial evaluating mobile text messaging to promote retention and adherence to antiretroviral therapy for people living with HIV in Burkina Faso. JMIR Res Protoc 2016;5:e170.

39. Elul B, Lamb MR, Lahuerta M, et al. A combination intervention strategy to improve linkage to and retention in HIV care following diagnosis in Mozambique: A clusterrandomized study. PLoS Med 2017;14:e1002433.*

40. Georgette N, Siedner MJ, Petty CR, Zanoni BC, Carpenter $\mathrm{S}$, Haberer JE. Impact of a clinical program using weekly Short Message Service (SMS) on antiretroviral therapy adherence support in South Africa: A retrospective cohort study. BMC Med Inf Decis Mak 2017;17:18.*

41. McNairy ML, Lamb MR, Gachuhi AB, et al. Effectiveness of a combination strategy for linkage and retention in adult HIV care in Swaziland: The Link4Health cluster randomized trial. PLoS Med 2017;14:e1002420.*

42. Orrell C, Cohen K, Mauff K, Bangsberg DR, Maartens G, Wood R. A randomized controlled trial of real-time electronic adherence monitoring with text message dosing reminders in people starting first-line antiretroviral therapy. J Acquir Immune Def Syndr 2015;70:495-502.*

43. Linnemayr S, Huang H, Luoto J, et al. Text messaging for improving antiretroviral therapy adherence: No effects after 1 year in a randomized controlled trial among adolescents and young adults. Am J Public Health 2017;107:1944-1950.*

44. Lester RT, Ritvo P, Mills EJ, et al. Effects of a mobile phone short message service on antiretroviral treatment adherence in Kenya (WelTel Kenya1): A randomised trial. Lancet 2010;376:1838-1845.*

45. Haberer JE, Musiimenta A, Atukunda EC, et al. Short message service (SMS) reminders and real-time adherence monitoring improve antiretroviral therapy adherence in rural Uganda. AIDS 2016;30:1295.*

46. Kassaye SG, Ong'ech J, Sirengo M, et al. Clusterrandomized controlled study of SMS text messages for prevention of mother-to-child transmission of HIV in rural Kenya. AIDS Res Treat.* 2016;2016:1289328.

47. Ruan Y, Xiao X, Chen J, Li X, Williams AB, Wang H. Acceptability and efficacy of interactive short message service intervention in improving HIV medication adher- ence in Chinese antiretroviral treatment-naïve individuals. Patient Prefer Adherence 2017;11:221.*

48. Davey DJ, Nhavoto JA, Augusto O, et al. SMSaude: Evaluating mobile phone text reminders to improve retention in HIV care for patients on antiretroviral therapy in Mozambique. J Acquir Immune Def Syndr 2016;73:e23.*

49. Maduka O, Tobin-West CI. Adherence counseling and reminder text messages improve uptake of antiretroviral therapy in a tertiary hospital in Nigeria. Niger J Clin Pract 2013;16:302-308.*

50. Sabin LL, DeSilva MB, Gill CJ, et al. Improving adherence to antiretroviral therapy with triggered real time text message reminders: The China through technology study (CATS). J Acquir Immune Def Syndr 2015;69:551.*

51. Marcolino MS, Oliveira JAQ, D’Agostino M, Ribeiro AL, Alkmim MBM, Novillo-Ortiz D. The impact of mHealth interventions: Systematic review of systematic reviews. JMIR Mhealth Uhealth 2018;6:e23.

52. Kanters S, Park JJ, Chan K, Socias ME, Ford N, Forrest JI. Interventions to improve adherence to antiretroviral therapy: A systematic review and network meta-analysis. Lancet HIV 2017;4:e31-e40.

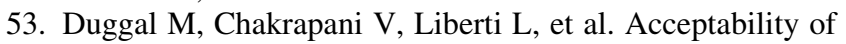
mobile phone-based nurse-delivered counseling intervention to improve HIV treatment adherence and self-care behaviors among HIV-positive women in India. AIDS Patient Care STDs 2018;32:349-359.

54. Sarabi RE, Sadoughi F, Orak RJ, Bahaadinbeigy K. The effectiveness of mobile phone text messaging in improving medication adherence for patients with chronic diseases: A systematic review. Iran Red Crescent Med J 2016;18: e25183.

55. Cooper V, Clatworthy J, Whetham J; EmERGE Consortium. mHealth interventions to support self-management in HIV: A systematic review. Open AIDS J 2017;11, 119132.

56. Dillingham R, Ingersoll K, Flickinger TE, et al. PositiveLinks: A mobile health intervention for retention in HIV care and clinical outcomes with 12-month follow-up. AIDS Patient Care STDs 2018;32:241-250.

57. Tanner AE, Song EY, Mann-Jackson L, et al. Preliminary impact of the weCare social media intervention to support health for young men who have sex with men and transgender women with HIV. AIDS Patient Care STDs 2018; $32: 450-458$.

58. Mimiaga MJ, Bogart LM, Thurston IB, et al. Positive Strategies to Enhance Problem-Solving Skills (STEPS): A pilot randomized, controlled trial of a multicomponent, technology-enhanced, customizable antiretroviral adherence intervention for HIV-infected adolescents and young adults. AIDS Patient Care STDs 2018;33:21-24.

59. Bigna JJR, Noubiap JJN, Kouanfack C, Plottel CS, KoullaShiro S. Effect of mobile phone reminders on follow-up medical care of children exposed to or infected with HIV in Cameroon (MORE CARE): A multicentre, single-blind, factorial, randomised controlled trial. Lancet Infect Dis 2014; 14:600-608.*

60. Kliner M, Knight A, Mamvura C, Wright J, Walley J. Using no-cost mobile phone reminders to improve attendance for HIV test results: A pilot study in rural Swaziland. Infect Dis Poverty 2013;2:12.*

61. Haberer JE, Kiwanuka J, Nansera D, et al. Real-time adherence monitoring of antiretroviral therapy among HIV- 
infected adults and children in rural Uganda. AIDS 2013; 27:2166-2188.*

62. Rodrigues R, Shet A, Antony J, et al. Supporting adherence to antiretroviral therapy with mobile phone reminders: Results from a cohort in South India. PLoS One 2012;7:e40723.*

63. Schwartz SR, Clouse K, Yende N, et al. Acceptability and feasibility of a mobile phone-based case management intervention to retain mothers and infants from an option $\mathrm{B}+$ program in postpartum HIV care. Matern Child Health J 2015;19:2029-2037.*

64. Huang D, Sangthong R, McNeil E, Chongsuvivatwong V, Zheng W, Yang X. Effects of a phone call intervention to promote adherence to antiretroviral therapy and quality of life of HIV/AIDS patients in Baoshan, China: a randomized controlled trial. AIDS Res Treat 2013.* DOI:10.1155/2013/ 580974
65. Uzma Q, Emmanuel F, Ather U, Zaman S. Efficacy of interventions for improving antiretroviral therapy adherence in HIV/AIDS cases at PIMS, Islamabad. J Int Assoc Physicians AIDS Care 2011;10:373-383.*

Address correspondence to: Natascha Wagner, PhD

Institute of Social Studies Erasmus University Rotterdam Kortenaerkade 12 2518AX The Hague The Netherlands

E-mail: wagner@iss.nl 